\title{
Validación y precisión de la escala de deterioro global (GDS) para establecer severidad de demencia en una población de Lima
}

\author{
Validation and diagnostic accuracy of the Global Deterioration \\ Scale (GDS) to establish severity of dementia in a population \\ of Lima
}

\begin{abstract}
Nilton Custodio' a,b \& RSG, Yahaira Becerra-Becerra ${ }^{2,4}$ c RDLYC, Carlos Alva-Diaz ${ }^{3,4}$ c RSG, Rosa Montesinos 5 d RSG, David Lira1 a,e RSG, Eder Herrera-Pérez ${ }^{6, c}$ RSG, José Cuenca-Alfaro ${ }^{7 \text { fSG }}$, Elizabeth Valeriano-Lorenzo7 f RDLYC, Sheila Castro-Suárez',8 a ORCID
\end{abstract}

Fecha correspondencia: Recibido: agosto 7 de 2016. Revisado: febrero 15 de 2017. Aceptado: marzo 3 de 2017.

Forma de citar:

Custodio N, Becerra-Becerra Y, Alva-Diaz C, Montesinos R, Lira D, Herrera-Pérez E, et al. Validación y precisión de la Escala de Deterioro Global (GDS) para establecer severidad de demencia en una población de Lima. Rev CES Med 2017; 31(1): 14-26.

\section{Open access}

(C) Derecho de autor

Licencia creative commons

Ética de publicaciones

Revisión por pares

Gestión por Open Journal System DOI: http://dx.doi.org/10.21615/ cesmedicina.31.1.2

ISSN 0120-8705

e-ISSN 2215-9177

\section{Comparte}

\section{F日G.}

\section{Resumen}

Objetivo: validar y evaluar el rendimiento de la Global Deterioration Scale (GDS) en una población que acude a una unidad especializada de Lima. Pacientes y métodos: investigación de tipo transversal con un diseño descriptivo comparativo. El estudio incluyó 215 individuos seleccionados de forma abierta, que acudieron a la unidad de diagnóstico de deterioro cognitivo y prevención de demencia. Se estudiaron tres grupos: 60 controles, 40 con diagnóstico de deterioro cognitivo leve y 115 con demencia. Los individuos fueron sometidos a evaluaciones sucesivas: cribado, diagnóstico y estadiaje de demencia y tipo de demencia. La validez y fiabilidad de la clasificación de severidad fue demostrada mediante la concordancia entre la medición del Global Deterioration Scale con el Clinical Dementia Rating (CDR). El rendimiento de Global Deterioration Scale se valoró mediante la obtención de los valores de sensibilidad y especificidad. Resultados: los promedios de edad fueron de 69, 70, y 74 años para los controles, los pacientes con deterioro cognitivo leve y los pacientes con demencia, respectivamente. La Global Deterioration Scale tuvo una buena correlación con Clinical Dementia Rating ( $r$ de Spearman =0,97; P=0,0001). La sensibilidad de la escala para establecer estadio de demencia fue de $79 \%$, con especificidad del $100 \%$ para cualquier categoría de demencia según los criterios diagnósticos estándares y según Clinical Dementia Rating. Conclusiones: se encontró una correlación casi perfecta entre Global Deterioration Scale y Clinical Dementia Rating; sin embargo, la Global Deterioration Scale presenta una sensibilidad moderada para establecer el estadio de demencia con respecto a Clinical Dementia Rating, mostrando además ser utilidad en el diagnóstico de deterioro cognitivo leve.

Palabras clave: Global Deterioration Scale, Clinical Dementia Rating, Demencia, Escalas de estadiaje clínico de demencia.

\section{Abstract}

Objective: To validate and evaluate diagnostic accuracy of the Global Deterioration Scale (GDS) in a population of a specialized unit of Lima. Patients 
Enero - Junio 2017 - Pág 15

\section{Sobre los autores:}

1 Servicio de Neurología, Instituto Peruano de Neurociencias. Lima, Perú. 2 Servicio de Geriatría, Hospital Militar Geriátrico. Lima, Perú. 3 Servicio de Neurología, Hospital Nacional Daniel Alcides Carrión. Callao, Perú. 4 Red de Eficacia Clínica y Sanitaria REDECS, Lima, Perú. 5 Unidad de Medicina de Rehabilitación, Instituto Peruano de Neurociencias. Lima, Perú. 6 Unidad de Proyectos de Investigación, Instituto de Salud del Niño. Lima, Perú. 7 Unidad de Neuropsicología, Instituto Peruano de Neurociencias. Lima, Perú. 8 Servicio de Neurología de la Conducta, Instituto Nacional de Ciencias Neurológicas. Lima, Perú.

a Médico Neurólogo;

b Magister en Neurociencias; c Médico cirujano; d Médico Rehabilitador; e Magister en salud pública, f Psicólogo.

En países en vías de desarrollo urge tener la capacidad discriminativa para detectar demencia en estadios iniciales y determinar el nivel de severidad, teniendo en cuenta el escaso tiempo disponible en los servicios de atención primaria de salud. and Methods: This research is a comparative cross-sectional descriptive design. The study included 215 individuals selected openly, who attending in the diagnostic unit of cognitive impairment and dementia prevention. Three groups were studied: 60 controls, 40 individuals diagnosed with mild cognitive impairment (MCl) and 115 with dementia diagnosis. They were assessments in three phases: screening, diagnosis and staging of dementia and type of dementia. The validity and reliability of the classification of severity was demonstrated by measuring the correlation between the GDS with the CDR (Clinical Dementia Rating). The diagnostic accuracy of GDS was evaluated by obtaining the values of sensitivity and specificity. Results: The average age was 69, 70, and 74 years for controls, patients with $\mathrm{MCl}$ and patients with dementia respectively. GDS had a good correlation with CDR (Spearman $r=0.97, P=0.0001$ ). The sensitivity of the GDS to set stage of dementia for any category of dementia was $79 \%$ and specificity of $100 \%$ according to standard diagnostic criteria and according to CDR. Conclusions: We demonstrated an almost perfect correlation between GDS and CDR, however GDS has a moderate sensitivity to set the stage dementia regarding CDR also proving to be useful in the diagnosis of $\mathrm{MCl}$.

Keywords: Global Deterioration Scale, Clinical Dementia Rating, Dementia, Scales clinical staging of dementia.

\section{Introducción}

La demencia no es una enfermedad, es un síndrome crónico que describe alteraciones adquiridas de la memoria y otras habilidades cognitivas, además de cambios en la conducta, pero que sobre todo compromete las actividades de vida diaria. Según progresa la disfunción cognitiva se incrementa también el deterioro de la funcionalidad (1). La prevalencia de la demencia en Latinoamérica, en comunidad de adultos mayores de 65 años de edad, es 7,1 \%, similar a la de los países desarrollados (2); mientras que en Perú es de $6,8 \%$, siendo la enfermedad de Alzheimer (EA), el tipo de demencia más frecuente (3).

En países en vías de desarrollo urge tener la capacidad discriminativa para detectar demencia en estadios iniciales y determinar el nivel de severidad, teniendo en cuenta el escaso tiempo disponible en los servicios de atención primaria de salud, por lo que se hace necesario contar con pruebas de cribado de rápida administración, alta sensibilidad y especificidad.

Las pruebas usualmente empleadas como el Mini-Mental State Examination (MMSE), muestran un efecto de techo, son poco sensibles a los cambios iniciales de la demencia y se ven afectadas por la edad y el nivel de instrucción del sujeto evaluado. En Lima, la prueba del dibujo del reloj de Manos (PDR-M) ha demostrado ser un instrumento breve y confiable para la detección de demencia, aunque también puede ser influenciada por el nivel de instrucción (4). El Addenbrookes's Cognitive Examination (ACE), versión en español, ha demostrado mejor sensibilidad que el Mini-Mental State Examination, pero requiere más tiempo y entrenamiento para su ejecución (ㅁ), mientras que el test de alteración de memoria (T@M), detecta deterioro cognitivo leve y enfermedad de Alzheimer en estadios iniciales, pero no ha sido evaluada en otros tipos de demencia ( $\underline{6})$.

Cuando se emplean evaluaciones basadas en el informante, éstas suelen ser más útiles para demostrar cambios cognitivos tempranos, comparadas con las evaluaciones objetivas breves basadas en el paciente, pues no están influenciadas por la edad, nivel de instrucción del paciente o tipo de cuidador. En ese sentido, las pruebas 
La Global Deterioration Scale (GDS) es una herramienta fácil de usar, puede ser empleada para evaluar quejas cognitivas subjetivas, es más detallada y clasifica los estadios de la demencia en siete. de cambios intra-individuales administradas a una fuente confiable, como la escala Clinical Dementia Rating (CDR), tienen mayor sensibilidad en la detección de cambios cognitivos que las pruebas cognitivas con normas inter-individuales, pero su aplicación requiere más tiempo y suficiente entrenamiento para la práctica clínica habitual. Habitualmente, esta escala es utilizada en casos de diagnósticos ya establecidos de demencia y es útil para determinar el nivel de severidad; siendo empleada ampliamente en estudios epidemiológicos.

La escala fue publicada en 1982 y aunque originalmente fue desarrollada para establecer el nivel de severidad de demencia en pacientes con enfermedad de Alzheimer, su uso se ha extendido a otros tipos de demencia (7).

La Clinical Dementia Rating es una entrevista semi-estructurada independiente que se realiza al paciente, aunque previamente se entrevista a un informante confiable (usualmente el cónyuge o un cuidador primario) para confirmar las habilidades cognitivas del paciente. Se considera una escala clínica global, pues también mide cambios sociales, conductuales y funcionales en el paciente evaluado. Entre sus ventajas están: es independiente de otras pruebas psicométricas, no requiere una evaluación basal y puede ser usada como control para cada individuo. Además, tiene buena confiabilidad inter-evaluador (ㅇ) , validez concurrente (9), validez predictiva (10) y correlación neuropatológica (11). Entre sus desventajas se citan: requiere entrenamiento especial, adecuadas habilidades y buen juicio del entrevistador para obtener información pertinente ya que toma por lo menos 30 minutos en ser administrada (12).

Por el contrario, la Global Deterioration Scale (GDS) es una herramienta fácil de usar, puede ser empleada para evaluar quejas cognitivas subjetivas, es más detallada y clasifica los estadios de la demencia en siete, mientras que el Clinical Dementia Rating lo hace en cinco. Su principal desventaja es que su sistema de puntuación no es sistemático y puede ser inexacta, por lo que no se recomienda en sistemas primarios de atención (13).

El objetivo del presente estudio fue la validación y evaluación del rendimiento de la Global Deterioration Scale en una población que acude a una unidad especializada de Lima.

\section{Metodología}

Diseño del estudio: investigación de tipo transversal con un diseño descriptivo comparativo.

Tests de estudio: la encuesta Clinical Dementia Rating (CDR) es una escala global de cinco puntos. Sus contenidos se dividen en seis ejes (memoria, orientación, juicio y solución de problemas, trabajo en la comunidad, rendimientos en casa y en aficiones y cuidado personal). La puntuación va de 0 a 3: estadio 0 (sujeto sano), 0,5 (demencia cuestionable), 1 (demencia leve), 2 (demencia moderada) y 3 (demencia severa) (12).

La Global Deterioration Scale es una escala que consiste en la descripción clínica de siete fases diferenciadas desde la normalidad hasta los grados más severos de la demencia de enfermedad de Alzheimer. Su puntuación es: estadio 1 (normal), estadio 2 (queja subjetiva de memoria), estadio 3 (deterioro cognitivo leve), estadio 4 (demencia leve), estadio 5 (demencia moderada), estadio 6 (demencia moderadamente severa) y estadio 7 (demencia severa) (14). 
Enero - Junio 2017 - Pág 17

Para comparar los resultados del GDS y CDR, el estándar de diagnóstico de demencia fue el resultado de la evaluación neuropsicológica, los resultados de los exámenes de sangre y de las neuroimágenes.
Los entrevistadores (10 psicólogos, estudiantes de postgrado en neuropsicología clínica) recibieron entrenamiento teórico y práctico en GDS y CRD durante cuatro horas semanales el primer mes, y en las cuales realizaron prácticas entre ellos, y luego durante dos horas semanales en el mes siguiente, realizaban entrenamiento con individuos sanos. Finalmente, en el último mes y durante dos horas diarias, tres veces por semana evaluaron pacientes con demencia y deterioro cognitivo leve. En esta etapa, de manera aleatoria para cada evaluador, se realizaba una filmación del procedimiento para determinar posibles errores. Antes de iniciar las evaluaciones del presente proyecto de investigación, los entrevistadores fueron evaluados para determinar la confianza inter-evaluador, el cual fue establecido como superior a 0,90.

Gold estándar: para comparar los resultados del GDS y CDR, el estándar de diagnóstico de demencia fue el resultado de la evaluación neuropsicológica, los resultados de los exámenes de sangre y de las neuroimágenes con los cuales se procedió a realizar el diagnóstico del tipo de demencia, mediante consenso entre neurólogos y neuropsicólogos del equipo. Los especialistas en neuropsicología que aplicaron las baterías neuropsicológicas desconocían los resultados de las pruebas cognitivas breves y de las escalas de severidad de demencia realizada en las fases previas. La evaluación neuropsicológica incluye una batería de pruebas cognitivas validadas para población peruana e incluyeron: Rey Auditory Verbal Learning Test, Logical Memory Subtest del Weschler Memory Scale revisado, Trail Making Test A y B, figura compleja de Rey, test de denominación de Boston, Wisconsin Card Sorting Test, Letter- Number (subtest del Weschler Adult Intelligent Scale III), Digit Span, copia de dibujos de Strub-Black, test de los cubos del WAIS-III. La batería neuropsiquiátrica estuvo constituida por el Neuropsychiatric Inventory (15) y la evaluación funcional según Alzheimer's Disease Cooperative Study - Activities of Daily Living (ADCS-ADL) (16).

Población y muestra: el estudio incluyó 215 individuos seleccionados de forma abierta que acudieron a la unidad de diagnóstico de deterioro cognitivo y prevención de demencia de la Clínica Internacional de Lima, entre enero del 2009 y diciembre del 2015. Se estudiaron tres grupos: 60 controles, 40 pacientes con diagnóstico de deterioro cognitivo leve (DCL) y 115 con diagnóstico de demencia.

Los criterios de inclusión para la muestra a estudiar fueron: edad superior a 60 años de edad, mantenerse cognitivamente sano o cumplir con los criterios diagnósticos de deterioro cognitivo leve o demencia basado en DSM IV (17), que incluyeron casos con diagnóstico de enfermedad de Alzheimer y demencia vascular (DV).

El diagnóstico de enfermedad de Alzheimer probable se realizó según criterios del National Institute for Communicative Disorders and Stroke-Alzheimer's Disease and Related Disorders Association (NINCDS-ADRDA) (18); el diagnóstico de demencia vascular según los criterios de National Institute of Neurological Disorders and Stroke and Association Internationale pour la Recherche' et l'Enseignement en Neurosciences (NINDS-AIREN) (19) y para deterioro cognitivo leve nos basamos en los criterios establecidos por Petersen (20). El grupo control estuvo conformado por familiares de los pacientes o voluntarios sanos, pareados por edad y sexo, sin quejas subjetivas de memoria.

Los criterios de exclusión fueron: individuos con dificultad para realizar las pruebas cognitivas debido a problemas auditivos, visuales u otros problemas físicos que pudieran interferir con su rendimiento; idioma diferente al español; bajo nivel de instrucción (definido como aquellos con menos de cuatro años de educación); 
Todos los individuos fueron sometidos a las siguientes evaluaciones sucesivas: cribado (fase 1), diagnóstico y estadio de demencia (fase 2), y tipo de demencia (fase 3). diagnóstico de depresión; antecedentes de adicción o abuso de sustancias; diagnóstico de demencia frontotemporal, asociada a enfermedad de Parkinson y con cuerpos de Lewy; deterioro cognitivo explicado por otra causa, (p.e. hipotiroidismo, deficiencia de vitamina B12, hepatopatía, nefropatía crónica, neuro-infecciones (infección asociada a VIH, sífilis), traumatismo cráneo encefálico severo, hematoma subdural, entre otras.

Procedimientos del estudio: todos los individuos fueron sometidos a las siguientes evaluaciones sucesivas: cribado (fase 1), diagnóstico y estadio de demencia (fase 2), y tipo de demencia (fase 3). Durante la fase de cribado (fase 1) fueron sometidos a una evaluación clínica integral y a pruebas cognitivas breves que incluyeron: MMSE (21), PDR-M (4) y el Pfeffer Functional Activities Questionnaire (PFAQ) (22).

Quienes tuvieron respuestas por debajo de los puntajes establecidos para esta investigación fueron sometidos a una segunda evaluación en la cual se realizaron nuevamente el MMSE y el PDR-M por un evaluador distinto al que realizó la fase de cribado. El punto de corte en el MMSE para sospecha de demencia fue ajustado según años de educación: 27 para individuos con más de siete años de educación, 23 para aquellos con cuatro a siete años de educación, 22 para aquellos con 1 a 3 años de educación y 18 para los iletrados (3).

La PDR-M evalúa la capacidad del individuo para poner sobre un círculo dibujado los números del 1 al 12, tal como van en un reloj y luego evalúa la dirección y proporcionalidad de las manecillas del reloj al intentar plasmar las 11:10 horas. El puntaje máximo es 10 y en individuos de Lima el puntaje menor a siete, indica compromiso cognitivo (4).

El PFAQ incluye 11 preguntas acerca de las actividades de la vida diaria, con rangos de puntaje de 0 a 3 según la severidad de la discapacidad en cada actividad. El puntaje máximo es 33 y el puntaje mayor de seis indica compromiso funcional (22).

Los individuos que confirmaron "deterioro cognitivo" en la segunda evaluación ingresaron a la fase de diagnóstico y estadio de demencia (fase 2), en la cual fueron sometidos a exámenes sanguíneos (niveles de hemoglobina, glucosa, urea, creatinina, pruebas de función hepática: TGO y TGP, niveles séricos de albúmina y globulina, medición de vitamina B12 y ácido fólico, perfil tiroideo: T3, T4 y TSH, niveles de electrolitos séricos: sodio, potasio y cloro; descarte de sífilis: VDRL y descarte de infección por VIH según prueba de Elisa), tomografía cerebral y/o resonancia magnética cerebral, evaluación de síntomas depresivos para descartar pseudo-demencia (inventario de depresión de Beck-BDI-II) y se aplicaron las escalas GDS y CDR. Los pacientes con un resultado de BDI-II mayor a 17, fueron excluidos para evitar la probabilidad de síntomas cognitivos de depresión. En esta fase aplicamos los criterios de demencia, basados en DSM-IV (17) y el estadio según CDR (12).

En la última fase (establecer tipo de demencia) se realizó la evaluación neuropsicológica en pacientes con deterioro cognitivo leve y demencia, con la finalidad de establecer los tipos específicos para cada uno. Aplicamos los criterios de National Institute for Communicative Disorders and Stroke-Alzheimer's Disease and Related Disorders Association (NINCDS-ADRDA) (18) para enfermedad de Alzheimer; los criterios de National Institute of Neurological Disorders and Stroke and Association Internationale pour la Recherche' et l'Enseignement en Neurosciences (NINDS-AIREN) (19) para demencia vascular y para deterioro cognitivo leve nos basamos en los criterios establecidos por Petersen (20). El grupo control estuvo conformado por familiares 
Enero - Junio 2017 - Pág 19

Los promedios del puntaje de MMSE en el grupo control, con deterioro cognitivo leve y demencia fueron de 25,$02 ; 21,36$ y 18,32 , respectivamente. de los pacientes o voluntarios sanos, pareados por edad y sexo, sin quejas subjetivas de memoria.

Métodos estadísticos: para realizar el análisis de los datos se utilizó el paquete estadístico STATA ${ }^{\circledR}$ versión 14 mediante la función diagti. Se analizaron los datos paramétricos con t de student o análisis de varianza de una vía (ANOVA). Se usaron pruebas no paramétricas (Mann-Whitney o Kruskall-Wallis, y correlación de Spearman) para datos ordinales y para aquellos sin distribución normal. Las variables categóricas fueron analizadas con pruebas de asociación (chi cuadrado con corrección de Yates o Fisher según fuera lo apropiado).

Para conocer el rendimiento de GDS y CDR se realizaron comparando cada una de ellas frente la prueba de oro, teniendo en cuenta la prevalencia de demencia de Lima $(6,85 \%)$. De esta manera, se consideraron los siguientes indicadores: sensibilidad (S), especificidad (E), valor predictivo positivo (VPP), valor predictivo negativo (VPN), área ROC (área bajo la receiver operating characteristic curve), cociente de probabilidad positivo (CPP) o Likehood Ratio positivo (LR +) y cociente de probabilidad negativo (CPN) o Likehood Ratio negativo (LR -).

La validez y fiabilidad de la clasificación de severidad fue demostrada con la concordancia entre la medición del GDS con el CDR y otras pruebas (porcentaje de concordancia, t y k de Kendall). Además, en todos los participantes del estudio obtuvimos la concordancia entre GDS y CDR, MMSE y ADCS-ADL.

Aspectos éticos: todos los participantes y sus cuidadores (cuando fue apropiado) firmaron un consentimiento informado de acuerdo con las guías éticas para investigación con sujetos humanos. El protocolo de estudio fue aprobado por la unidad de investigación de la Clínica Internacional y la Universidad de San Martin de Porres de la ciudad de Lima.

\section{Resultados}

El promedio de edad de los controles fue de 69,4 años, mientras que el de los pacientes con deterioro cognitivo leve fue de 70,2 y el de los pacientes con demencia de 74,1 años, encontrándose diferencias entre los controles y los pacientes con demencia. Se observó un mayor porcentaje de mujeres en los tres grupos, sin diferencias entre los grupos.

Los promedios del puntaje de MMSE en el grupo control, con deterioro cognitivo leve y demencia fueron de 25,02; 21,36 y 18,32, respectivamente, encontrándose diferencias entre los tres grupos. En el puntaje obtenido en la prueba de dibujo del reloj-versión de manos se obtuvo una media de 8,92 para los controles, 6,13 para los pacientes con deterioro cognitivo leve y 3,92 para los pacientes con demencia, con diferencias entre el grupo control y el grupo con demencia.

La media del puntaje obtenido para el PFAQ fue de 1,54; 3,62 y 17,42, para los tres grupos, respectivamente; con diferencias entre el grupo control y el grupo con demencia. En relación a las escalas de severidad, encontramos que para la GDS los controles obtuvieron un puntaje promedio de $2,23 \pm 0,41$, mientras que el grupo con deterioro cognitivo breve obtuvo una media de 3,32 $\pm 0,56$ y el grupo de pacientes con demencia una media de 4,94, $\pm 0,72$; con diferencias entre los tres grupos. 
No se encontró correlación de la escala GDS con educación ( $r$ de Spearman $=0,07 ; \quad P=0,204)$ y edad ( $r$ de Spearman =0,04; $\mathrm{P}=0,47$ ); pero sí con Mini Mental State Examination ( $r$ de Spearman $=-0,77$; $P=0,0001)$ y CDR.
Para la segunda escala de severidad evaluada, la CDR, encontramos una media de 0,$46 ; 0,75$ y 1,79 para los tres grupos respectivamente; con diferencias entre los tres grupos (cuadro 1).

Cuadro 1. Perfil demográfico y rendimiento en pruebas cognitivas breves y escalas de severidad en una muestra de pacientes de una unidad especializada de Lima

\begin{tabular}{|c|c|c|c|c|}
\hline & $\begin{array}{l}\text { Controles } \\
(n=60)\end{array}$ & $\begin{array}{c}\text { Deterioro cognitivo } \\
\text { leve }(n=40)\end{array}$ & $\begin{array}{l}\text { Demencia } \\
(n=115)\end{array}$ & $P$ \\
\hline Edad & $69,4(3,9)$ & $70,2(3,8)$ & $74,1(3,7)$ & $0,001+$ \\
\hline Sexo femenino, $n$ (\%) & $35(58,3)$ & $25(62,5)$ & $70(60,8)$ & 0.265 \\
\hline Educación (años) & $11,1(2,6)$ & $12,4(2,7)$ & $12,2(2,4)$ & 0,396 \\
\hline MMSE & $25,02(1,26)$ & $21,36(1,98)$ & $18,32(2,78)$ & $0,001 * \dagger$ \\
\hline PDR-M & $8,92(0,83)$ & $6,13(2,92)$ & $3,92(2,71)$ & $0,0001+$ \\
\hline PFAQ & $1,54(1,66)$ & $3,62(1,45)$ & $17,42(3,56)$ & $0,0001+$ \\
\hline GDS & $2,23(0,41)$ & $3,32(0,56)$ & $4,94(0,72)$ & $0,001 * \dagger$ \\
\hline CDR & $0,46(0,23)$ & $0,75(0,32)$ & $1,79(0,76)$ & $0,001 * \dagger$ \\
\hline
\end{tabular}

${ }^{*}$ Control vs deterioro cognitive leve

† Control vs demencia

MMSE: Mini Mental State Examination; PDR-M: Prueba de Dibujo del Reloj-versión de Manos; PFAQ: Pfeffer Functional

Activities Questionnaire; GDS: Global Deterioration Scale; CDR: Clinical Dementia Rating.

No se encontró correlación de la escala GDS con educación ( $r$ de Spearman =0,07; $P=0,204$ ) y edad ( $r$ de Spearman $=0,04 ; P=0,47$ ); pero sí con Mini Mental State Examination ( $r$ de Spearman $=-0,77 ; P=0,0001$ ) y CDR ( $r$ de Spearman $=0,97 ; P=0,0001$ ). El MMSE fue significativamente diferente entre todas las categoría del Global Deterioration Scale (ANOVA de una vía, $\mathrm{F}=135,11 ; \mathrm{P}=0,0001$ ) evidenciando una correcta clasificación de severidad del mismo. Ello también se observó con las pruebas de neuropsicología y una clara disminución de los puntajes con el incremento del Global Deterioration Scale (datos no mostrados).

La consistencia interna del GDS fue muy buena (alfa de Cronbach =0,82). La validez convergente fue medida al comparar el rendimiento del GDS respecto al MMSE, CDR y ADCS-ADL. Los coeficientes de correlación de los puntajes del GDS en los grupos de estudio estuvieron en rangos de $-0,72$ a 0,81 (cuadro 2).

Cuadro 2. Validez concurrente de GDS en una muestra de pacientes de una unidad especializada de Lima

\begin{tabular}{lcccc}
\hline & $\begin{array}{c}\text { Controles } \\
(\mathbf{n}=\mathbf{6 0})^{*}\end{array}$ & $\begin{array}{c}\text { Deterioro cognitivo leve } \\
(\boldsymbol{n}=\mathbf{4 0})^{*}\end{array}$ & $\begin{array}{c}\text { Demencia } \\
(\boldsymbol{n}=\mathbf{1 1 5})^{*}\end{array}$ & $\boldsymbol{P}$ \\
\hline GDS vs MMSE & $-0,72$ & $-0,61$ & $-0,48$ & $<0,001$ \\
GDS vs CDR & 0,81 & 0,75 & 0.69 & $<0,001$ \\
GDS vs ADCS-ADL & $-0,77$ & $-0,73$ & 0.70 & $<0,001$ \\
\hline
\end{tabular}

* Coeficientes de correlación por Correlación de Pearson

GDS: Global Deterioration Scale; MMSE: Mini Mental State Examination; CDR: Clinical Dementia Rating; ADCS-ADL: Alzheimer's Disease Cooperative Study - Activities of Daily Living 
Enero - Junio 2017 - Pág 21

La sensibilidad del Global Deterioration Scale para establecer estadio de demencia fue de $79 \%$, con especificidad del $100 \%$ para cualquier categoría de demencia.
La sensibilidad del Global Deterioration Scale para establecer estadio de demencia fue de $79 \%$, con especificidad del $100 \%$ para cualquier categoría de demencia según los criterios diagnósticos estándares y según el Clinical Dementia Rating. Además GDS presentó un VPP de 41,8 \%, un VPN de 96,8 \%, un área ROC de 0,82, un CPP de 5,0 y un CPN de 0,2. Mientras que CDR presentó una sensibilidad de $89,3 \%$, una especificidad de $98,1 \%$, un VPP de 80,5\%, un VPN de $99.2 \%$, un área ROC de 0,94, un CPP o LR (+) de 48,8, un CPN o LR (-) de 0,11 (cuadro 3).

Cuadro 3. Rendimiento de GDS y CDR en una muestra de pacientes de una unidad especializada de Lima

\begin{tabular}{lcc}
\hline Indicador & GDS & CDR \\
\hline & $\%$ & $\%$ \\
Sensibilidad & $(I C 95 \%)$ & $(I C 95 \%)$ \\
Especificidad & 79,0 & 89,3 \\
& $(56,9-91,2)$ & $(81,8-92,7)$ \\
VPP & 100,0 & 98,1 \\
VPN & $(98,9-100)$ & $(93,4-98,9)$ \\
Área ROC & 41,8 & 80,5 \\
& $(28,5-52,3)$ & $(50,1-92,6)$ \\
LR + & 96,8 & 99,2 \\
& $(90,9-98,1)$ & $(98,3-99,8)$ \\
LR - & 0,82 & 0,94 \\
& $(3,71-0,93)$ & $(0,90-0,97)$ \\
\hline
\end{tabular}

GDS: Global Deterioration Scale; CDR: Clinical Dementia Rating; IC 95 \%: Intervalo de confianza al $95 \%$; VPP: Valor predictivo positivo; VPN: Valor predictivo negativo; Área ROC: área bajo la receiver operating characteristic curve; LR +: cociente de probabilidad positivo o Likehood Ratio positivo; LR -: cociente de probabilidad negativo (CPP) o Likehood Ratio negativo.

La Global Deterioration Scale fue capaz de establecer correctamente 16 de 17 pacientes calificados por CDR en estadio leve. Por otro lado, la GDS en cinco de 81 casos no pudo establecer el mismo estadio en pacientes calificados por Clinical Dementia Rating como estadio moderado (cuadro 4).

Cuadro 4. Severidad de demencia, comparando GDS vs CDR en una muestra de 115 pacientes con demencia de una unidad especializada de Lima

Clinical Dementia Rating (CDR)

\begin{tabular}{lccc} 
Global Deterioration Scale (GDS) & $\begin{array}{c}\mathbf{1} \\
\text { (demencia leve) } \\
\mathbf{( 1 7 )}\end{array}$ & $\begin{array}{c}\mathbf{2} \\
\text { (demencia } \\
\text { moderada) } \\
\mathbf{( 8 1 )}\end{array}$ & $\begin{array}{c}\mathbf{3} \\
\text { (demencia severa) } \\
\mathbf{( 1 7 )}\end{array}$ \\
\hline Leve (GDS 4) & $16(94,1 \%)$ & & \\
Moderado (GDS 5) & & $31(38,3 \%)$ & \\
Moderadamente severo (GDS 6) & & $45(93,7 \%)$ & \\
Severo (GDS 7) & & & $17(100 \%)$ \\
\hline$\chi^{2}-3884: P 00001$ & &
\end{tabular}


Conocer la severidad del compromiso cognitivo de un paciente es de utilidad para tomar decisiones terapéuticas, seleccionar fármacos aprobados por nivel de severidad de la demencia y uniformizar definiciones operacionales
El porcentaje de concordancia de GDS con todos los estadios de demencia según CDR fue de $85 \%, k=0,73(0,68-0,81)$ y $t=0,78(0,71-0,83)$; leve versus moderado fue $82 \%, k=0,77(0,69-0,80)$ y $t=0,80(0,75-0,86)$; y moderado versus severo, fue $86 \%$, $k=0,80(0,73-0,85)$ y $t=0,83(0,76-0,85)$.

\section{Discusión}

Conocer la severidad del compromiso cognitivo de un paciente es de utilidad para tomar decisiones terapéuticas, seleccionar fármacos aprobados por nivel de severidad de la demencia y uniformizar definiciones operacionales $(\underline{23}, 24)$. Dentro de estas escalas las más ampliamente utilizadas son Global Deterioration Scale y Clinical Dementia Rating (ㅁ, 25).

En el presente trabajo estudiamos tres grupos y en todos ellos el promedio de edad estuvo por encima de 65,5 años de edad; observándose que a mayor compromiso cognitivo y funcional mayor edad del paciente; además el género femenino predominó en todos los grupos; estos hallazgos son similares a los obtenidos en otros estudios en los que se ha investigado estos factores y se les considera como un factor de riesgo para demencia (26).

Llama la atención los valores promedios obtenidos luego de la evaluación con el MMSE en el grupo control $(1, \underline{26})$, a pesar de que el grado de instrucción de los participantes se encuentra por encima de once años de estudio y no tuvieron evidencia de compromiso cognitivo por las otras pruebas aplicadas. Al respecto, Custodio et al. encuentran baja sensibilidad de MMSE como prueba cognitiva breve (27).

Tanto los valores promedios obtenidos en el MMSE y la PDR-M en los grupos con DCL y demencia se encontraron por debajo de sus puntos de corte para disfunción cognitiva (4). El GDS en el grupo de control obtuvo un puntaje promedio de 2,23 que correspondería a pacientes con queja subjetiva de memoria y el CDR de 0,46 $(0,5)$ que se catalogaría como demencia incierta $(\underline{7}, \underline{25})$, a pesar de ser el grupo sin compromiso cognitivo. Quizá esto se deba a que estos valores no están validados en nuestra población o a que los evaluadores necesitaron mayor entrenamiento en la aplicación del CDR.

A este respecto, se ha observado que el $76 \%$ de evaluadores experimentados logran clasificar correctamente a sujetos en estadio 0 de CDR y que sólo $50 \%$ de estos evaluadores logran hacerlo correctamente a sujetos en el estadio 0,5 (28). Sin embargo, los valores promedios de GDS en el DCL y demencia sí corresponden la clasificación propuesta en los estudios originales de esta escala. En el caso del CDR, el tratar de hacer esta correlación resulta más difícil considerando los estadios de demencia por encima de 1, lo cual en nuestro estudio englobaría también al DCL ().

Nosotros correlacionamos el puntaje del GDS con el MMSE, CDR y el ADC-ADL, obteniéndose una buena correlación con el MMSE y el CDR. Son muy escasos los estudios que buscan esta correlación; así Choi et al. (29), realizan un estudio de intercambio de puntaje entre CDR y GDS y encuentran una correlación casi perfecta entre ambas escalas, de 0,97 para todos los sujetos, y de 0,97 y 0,96 para los subgrupos de sujetos sin demencia y enfermedad de Alzheimer y sujetos sin demencia y demencia vascular, respectivamente. Encontramos igualmente una correlación casi perfecta $(0,97)$ para todos los casos de demencia, coincidiendo con estos autores. 
Enero - Junio 2017 - Pág 23

En este caso los resultados de GDS no solo se podrían utilizar como escala de severidad sino también como test diagnóstico de deterioro cognitivo leve y/o demencia, ya que dentro de los ítems que evalúa figuran dominios cognitivos, de funcionalidad y síntomas psiquiátricos.
Así mismo, los mismos autores correlacionan MMSE con GDS encontrando una correlación de 0,95 para todos los sujetos, 0,95 y 0,91 para enfermedad de Alzheimer y demencia vascular, respectivamente. En nuestro estudio la correlación para todos los casos de demencia fue de 0,48. Estas diferencias en la correlación entre GDS y CDR pueden deberse a que el análisis de los datos difiere en la conformación de los grupos a comparar; igualmente, podría reflejar la necesidad de un mayor entrenamiento en la aplicación de CDR por parte de los evaluadores.

Encontramos estudios que correlacionan el puntaje del MMSE y el CDR (escala de severidad de uso similar al GDS) donde los autores concluyen que el MMSE es un test cognitivo también útil para la estatificación de la demencia; no obstante ambos test tienen dificultades para la discriminación en pacientes con demencia cuestionable o sin deterioro cognitivo (24).

Macedo y Ramos encuentran que casi la mitad de los casos considerados normales por MMSE resultaron casos cuestionables por CDR (30); Perneczky et al. reportan que la correlación entre los puntajes de MMSE y CDR es mejor conforme avanza la enfermedad pero a partir de DCL, pues la correlación para los casos indeterminados es pobre (24).

En este caso los resultados de GDS no solo se podrían utilizar como escala de severidad sino también como test diagnóstico de deterioro cognitivo leve y/o demencia, ya que dentro de los ítems que evalúa figuran dominios cognitivos, de funcionalidad y síntomas psiquiátricos (31).

La concordancia entre los estadios del GDS y el CDR fue buena en estados leves y severos de demencia, pese a ello se encontraron dificultades en la concordancia en estadios moderados. Estos datos demuestran que el CDR y el GDS discriminan pacientes con DCL y demencia severa.

Entre las limitaciones del estudio podemos mencionar que la muestra incluyó sólo dos tipos de demencia, como enfermedad de Alzheimer y demencia vascular; sin embargo, se justifica debido a que las guías de recomendaciones en otros tipos de demencia, como la fronto-temporal recomiendan la Fronto-Temporal Dementia Rating Scale (FRS) que fue desarrollada específicamente para demencia fronto-temporal, variante conductual (32). Otra limitación del estudio se refiere al bajo tamaño de muestra y a la escasa proporción de pacientes con CDR 1 y CDR 3.

\section{Conclusiones}

Teniendo en cuenta que las escalas de valoración global tienen evidentes ventajas sobre las psicométricas, como el tener la capacidad de identificar un mayor rango de severidad, estar menos influenciadas por el grado de instrucción, los factores culturales y lingüísticos, y tomando en cuenta la realidad de nuestra región, es de suma importancia utilizar test validados en nuestro medio que permitan cumplir esta tarea.

\section{Conflictos de intereses:}

Ninguno.

\section{Bibliografía}

1. Wimo A, Prince M: World Alzheimer Report 2010: The Global Economic Impact of Dementia. Alzheimer's Disease International: London, UK; 2010. https://www. alz.org/documents/national/World Alzheimer Report 2010.pdf 
2. Nitrini R, Bottino C, Albala C, Custodio N, et al. Prevalence of dementia in Latin American: a collaborative study of population-based cohorts. International Psychogeriatrics 2009;21:622-630. https://www.ncbi.nlm.nih.gov/pubmed/19505354

3. Custodio N, Garcia A, Montesinos R, Escobar J, et al. Prevalencia de demencia en una población urbana de Lima-Perú: Un estudio puerta a puerta. An Fac Med 2008:69(4):233-238. http://revistasinvestigacion.unmsm.edu.pe/index.php/anales/article/view/1110

4. Custodio N, Garcia A, Montesinos R, Lira D, et al. Validación de la prueba de dibujo del reloj-versión de Manos como prueba de cribado para detector demencia en una población adulta mayor de Lima, Perú. Rev Peru Med Exp Saud Publica 2011;28(1):29-34. http://www.scielosp.org/scielo.php?script=sci arttext\&pi$\underline{d}=\mathrm{S} 1726-46342011000100005$

5. Custodio N, Lira D, Montesinos R, Gleichgerrcht E, et al. Utilidad del Addenbrookes's Cognitive Examination versión en español en pacientes peruanos con enfermedad de Alzheimer y demencia frontotemporal. Vertex Rev Arg de Psiquiat 2012;XXIII:165-172.

6. Custodio N, Lira D, Herrera-Perez E, Nuñez del Prado L, Parodi J, Guevara-Silva E, et al. The Memory Alteration Test discriminates between Cognitively Healthy Status, Mild Cognitive Impairment and Alzheimer's Disease. Dementia Geriatric and Cognitive Disorder Extra 2014;4:314-321. https://www.ncbi.nlm.nih.gov/ pmc/articles/PMC4176469/

7. Hughes CP, Berg L, Danziger WL, Coben LA, et al. A new clinical scale for the staging of dementia. Br J Psychiatry 1982;140:566-572. https://www.ncbi.nlm.nih. gov/pubmed/7104545

8. Burke WJ, Miller JP, Rubin EH, Morris JC, et al. Reliability of the Washington University Clinical Dementia Rating. Arch Neurol 1988;45:31-32. https://www.ncbi. nlm.nih.gov/pubmed/3337672

9. Welsh KA, Butters N, Hughes JP, Mohs RC, et al. Detection and staging of dementia in Alzheimer's disease. Use of the neuropsychological measures developed for the Consortium to Establish a Registry for Alzheimer's Disease. Arch Neurol 1992;49:448-452. https://www.ncbi.nlm.nih.gov/pubmed/1580805

10. Berg L, Danziger WL, Storandt M, Coben LA, et al. Predictive features in mild senile dementia of the Alzheimer type. Neurology 1984;34:563-569. https://www. ncbi.nlm.nih.gov/pubmed/6538648

11. Berg L, McKeel DW Jr, Miller JP, Storandt M, et al. Clinicopathologic studies in cognitively healthy aging and Alzheimer's disease: relation of histologic markers to dementia severity, age, sex, and apolipoprotein E genotype. Arch Neurol 1998;55:326-335. https://www.ncbi.nlm.nih.gov/pubmed/9520006

12. Morris JC, Ernesto C, Schafer K, et al. Clinical dementia rating training and reliability in multicenter studies: the Alzheimer's Disease Cooperative Study experience. Neurology 1997;48:1508-1510. https://www.ncbi.nlm.nih.gov/pubmed/9191756 
13. Choi YJ, Won CW, Kim S, Choi HR, Kim BS, Jeon SY, et al., Five items differentiate mild to severe dementia from normal to minimal cognitive impairment using the Global Deterioration Scale. Journal of Clinical Gerontology \& Geriatrics (2015). http://www.sciencedirect.com/science/article/pii/S2210833515000684

14. Reisberg B, Ferris SH, Franssen E. An ordinal functional assessment tool for Alzheimer's type dementia. Hosp Comm Psychiat 1985; 36: 593-595. https://www. ncbi.nlm.nih.gov/pubmed/4007814

15. Cummings JL, Mega M, Gray K, Rosenberg-Thompson S, Carusi DA, Gornbein J. The Neuropsychiatric Inventory: comprehensive assessment of psychopathology in dementia. Neurology. 1994;44:2308-2314. https://www.ncbi.nlm.nih.gov/ pubmed/7991117

16. Galasko D, Bennett D, Sano M, Ernesto C, Thomas R, Grundman M, et al. An inventory to assess activities of daily living for clinical trials in Alzheimer's disease: the Alzheimer's Disease Cooperative Study. Alzheimer Dis Assoc Disord. 1997;11(Suppl 2):S33-S39. https://www.ncbi.nlm.nih.gov/pubmed/9236950

17. American Psychiatric Association. Diagnostic and statistical manual of mental disorders, DSM-IV-TR. 4th ed. Washington, DC: American Psychiatric Association, 2000.

18. McKhann G, Drachman D, Folstein M, Katzman R, Price D, Stadlan EM. Clinical diagnosis of Alzheimer's disease: report of the NINCDS-ADRDA work group under the auspices of department of health and human services task force on Alzheimer's disease. Neurology 1984;34:939-944. https://www.ncbi.nlm.nih.gov/ pubmed/6610841

19. Roman GC, Tatemichi TK, Erkinjuntti T, Cummings JL, Masdeu JC, Garcia JH. Vascular dementia: diagnostic criteria for research studies. Report of the NINDS-AIREN international workshop. Neurology. 1993;43:250-260. https://www.ncbi. nlm.nih.gov/pubmed/8094895

20. Petersen RC, Smith GE, Waring SC, Ivnik RJ, Tangalos EG \& Kokmen E. Mild cognitive impairment: clinical characterization and outcome. Arch Neurol. 1999;56:303308. https://www.ncbi.nlm.nih.gov/pubmed/10190820

21. Custodio N, Lira D. Adaptación Peruana del Mini Mental State Examination (MMSE). An Fac med. 2014; 75(1): 69. http://revistasinvestigacion.unmsm.edu. pe/index.php/anales/article/view/6951/6158

22. Quiroga P, Albala C and Klaasen G. Validación de un test de tamizaje para el diagnóstico de demencia asociado a edad, en Chile. Rev Med Chile 2004;132:467-478. $\quad$ http://www.scielo.cl/scielo.php?script=sci arttext\&pi$\underline{d}=50034-98872004000400009$

23. O'Bryant SE, Waring SC, Cullum CM, Hall J, Lacritz L, Massman PJ, Lupo PJ, Reisch JS, Doody R; Texas Alzheimer's Research Consortium. Staging dementia using Clinical Dementia Rating Scale Sum of Boxes scores: a Texas Alzheimer's research consortium study. Arch Neurol. 2008 Aug;65(8):1091-5. https://www. ncbi.nlm.nih.gov/pmc/articles/PMC3409562/ 
24. Perneczky R, Wagenpfeil S, Komossa K, Grimmer T, Diehl J, Kurz A. Mapping scores onto stages: mini-mental state examination and clinical dementia rating. Am J Geriatr Psychiatry. 2006 Feb;14(2):139-144. https://www.ncbi.nlm.nih.gov/ pubmed/16473978

25. Auer S1, Reisberg B. The GDS/FAST staging system. Int Psychogeriatr. 1997;9 Suppl 1:167-71. https://www.ncbi.nlm.nih.gov/pubmed/9447440

26. Wen YH1, Wu SS, Lin CH, Tsai JH, Yang P, Chang YP, Tseng KH. A Bayesian Approach to Identifying New Risk Factors for Dementia: A Nationwide Population-Based Study. Medicine (Baltimore). 2016 May;95(21):e3658 https://www.ncbi.nlm.nih. gov/pubmed/27227925

27. Custodio N, Alva-Diaz C, Becerra-Becerra Y, Montesinos R, Lira D, Herrera-Pérez $\mathrm{E}$, et al. Rendimiento de pruebas cognitivas breves en una muestra de pacientes viviendo en comunidad con demencia en estadios avanzados del Cercado de Lima. Rev Peru Med Exp Saud Publica (in press). https://www.researchgate.net/ publication/311714575 Rendimiento en pruebas cognitivas breves de adultos mayores con demencia en estadios avanzados residentes de una comunidad urbana de Lima Peru

28. Tractenberg RE, Schafer K, Morris JC. Interobserver disagreements on clinical dementia rating assessment: interpretation and implications for training. Alzheimer Dis Assoc Disord. 2001;15:155-161. https://www.ncbi.nlm.nih.gov/pub$\mathrm{med} / 11522933$

29. Choi SH, Lee BH, Kim S, Hahm DS, Jeong JH, Yoon SJ, Jeong Y, Ha CK, Nab DL. Interchanging scores between clinical dementia rating scale and global deterioration scale. Alzheimer Dis Assoc Disord. 2003 Apr-Jun;17(2):98-105. https:// www.ncbi.nlm.nih.gov/pubmed/12794387

30. Macedo M, Ramos L. Validity of the Portuguese version of Clinical Dementia Rating. Rev Saudé Pública. 2005; 39(6):1-5. http://www.scielo.br/scielo.php?pi$\mathrm{d}=\mathrm{s} 0034-89102005000600007 \&$ script $=$ sci arttext\&tlng=en

31. Eisdorfer C1, Cohen D, Paveza GJ, Ashford JW, Luchins DJ, Gorelick PB, Hirschman RS, Freels SA, Levy PS, Semla TP, et al. An empirical evaluation of the Global Deterioration Scale for staging Alzheimer's disease. Am J Psychiatry. 1992 Feb;149(2):190-194. https://www.ncbi.nlm.nih.gov/pubmed/1734738

32. Mioshi E, Hsieh S, Savage S, et al. Clinical staging and disease progression in frontotemporal dementia. Neurology 2010; 74: 1591-97. https://www.ncbi.nlm. nih.gov/pubmed/20479357 